\title{
El proceso de innovación dentro del diseño estratégico de las organizaciones
}

\begin{abstract}
RESUMEN
La innovación es el proceso que permite la creación de ventajas competitivas en las organizaciones. Forma parte de la estrategia empresarial como fuente de competitividad y diferenciación. La variable de innovación se incorpora al diseño estratégico en un proceso iterativo e incremental que integra la estrategia de innovación dentro de la estrategia de desarrollo de la empresa.

Este artículo describe los componentes del modelo de innovación que enlaza las posibilidades de cambio con la oportunidad de cubrir necesidades insatisfechas.
\end{abstract}

Palabras clave: Innovación, empresa, diseño estratégico, modelo, éxito.

THE PROCESSS OF INNOVATION IN THE STRATEGIC DESIGN OF THE ORGANIZATIONS

\section{ABSTRACT}

Innovation is the process that allows the creation of competitive advantage in organizations, It is part of the business strategy as source of competitiveness and differentiation. The variable of innovation is incorporated into the strategic design in an iterative and incremental process that integrates innovation strategy within development strategy of the company.

This article describes the components of the innovation model that links the possibilities for change with the opportunity to meet needs not satisfied.

Key words: Innovation, enterprise, strategic design, model, success.

\section{EL CONCEPTO DE INNOVACIÓN EN LAS ORGANIZACIONES}

Todas las organizaciones, llámense empresa, institución, ministerio, escuela, u otro, ofrece productos y servicios, donde el producto es un bien tangible real perceptible y el servicio es un intangible, una promesa de beneficio futuro. No existen sectores productivos o de servicios totalmente separados; así, se aplica el concepto de producto ampliado, donde el producto/servicio es el paquete de beneficios con valor para el cliente.

\section{¿Qué es innovación?}

Innovación es el proceso en el cual a partir de una idea, invención, o el reconocimiento de una necesidad, se desarrolla un producto, técnica o servicio útil, que se brinda comercialmente y es aceptado en el mercado. La innovación se sustenta en el conocimiento científico y empírico (Kuhn, 1962), se denominará tecnológica cuando se relacione con la ciencia y tecnología, lo que representa el elemento clave de la competitividad (Figura N. ${ }^{\circ}$ 1).

\section{Figura 1. Ciencia y Tecnología}

\begin{tabular}{|l} 
Ciencia U Tecnología \\
Ciencia $=$ e + Ic + Cc + Rc \\
$\mathrm{e}=$ enigma \\
$\mathrm{Ic}=$ investigación científica \\
$\mathrm{C} \mathrm{c}=$ conocimiento científico \\
$\mathrm{RC}=$ revoluciones científicas \\
Teconología $=$ la $+\mathrm{D}+\mathrm{i}+\mathbf{A}$ \\
la $=$ investigación aplicada \\
$\mathrm{D}=$ desarrollo tecnológico \\
$\mathrm{i}=$ innovación \\
$\mathrm{A}=$ asimilación o aprendizaje social
\end{tabular}

FUENTE: Elaboración propia.

La competitividad de una nación depende de la capacidad de su industria para innovar y mejorar (Porter, 1999) y la innovación se logra cuando el nuevo producto tiene éxito comercial y es competitivo (Escorsa-Valls, 2001).

\footnotetext{
Magíster en Administración, Ingeniero Industrial, Economista. Profesor en la Facultad de Ingeniería Industrial, Departamento de Producción y Gestión Industrial de la UNMSM. Email: aacevedo@speedy.com.pe

2 Magíster en Dirección por la Universidad de Piura, Ingeniero Industrial, estudios de Derecho. Profesora en la Facultad de Ingeniería de Sistemas e Informática de la UNMSM. Email: klinares@speedy.com.pe
} 


\section{Las tendencias del entorno de innovación}

Toda organización, para sobrevivir, mantenerse y crecer, debe implantar el criterio de innovación dentro del diseño de su estrategia de largo plazo. Las razones son:

- Globalización de la economía e intensificación de la competencia mundial.

- Masificación de mercados con personalización y sofisticación de segmentos.

- Explosión del conocimiento científico y del progreso técnico.

- Tecnologías de ruptura y surgimiento de nuevos sectores y modelos de negocio.

- Cambios de organización industrial y estrategias con ciclos de vida acelerados.

\section{La empresa productiva y de servicios}

Las actividades empresariales se orientaban a las tareas de producción, paulatinamente los servicios han elevado su peso de manera que las organizaciones, ahora, se orientan a las funciones de operación de servicios. Ya se percibe que la clasificación de empresa productiva o empresa de servicios, se ha indiferenciado y las fronteras son más y más difusas.

En el futuro, las empresas de éxito han de poseer una parte de producción y una parte de servicios, donde el producto empleará estrategias de liderazgo en costo y el servicio será diferenciador y orientado a los mercados.

\section{La empresa innovadora}

Innovación es un nuevo producto, nuevo proceso o nuevo servicio que proporciona utilidad y permite mejoras en la calidad de vida. Empresa innovadora es aquella que emplea el conocimiento existente para generar cambio y evolución, crea el know-how, realiza cosas nuevas, adopta y pone a punto nuevos procesos de fabricación de productos o servicios (Figura N. ${ }^{\circ} 2$ ). En el proceso de innovación intervienen diversas actividades científicas, que se plasman en la tecnología: equipamiento físico, procesos productivos, organización, finanzas y mercadeo.

\section{LA INNOVACIÓN DENTRO DEL DISEÑO ESTRATÉGICO}

La innovación es un proceso sistémico que se integra a la estrategia de las organizaciones. En la empresa, el proceso de administración estratégica enlaza el diseño de productos y servicios con el planeamiento general, alineando la misión y estrategia con las capacidades técnicas para desarrollar nuevas formas de cubrir las necesidades insatisfechas de los clientes.

Tradicionalmente, se ha considerado que la innovación es un proceso irregular, fruto de una idea ilumi-

Figura N. ${ }^{\circ}$. Ciencia y Tecnología

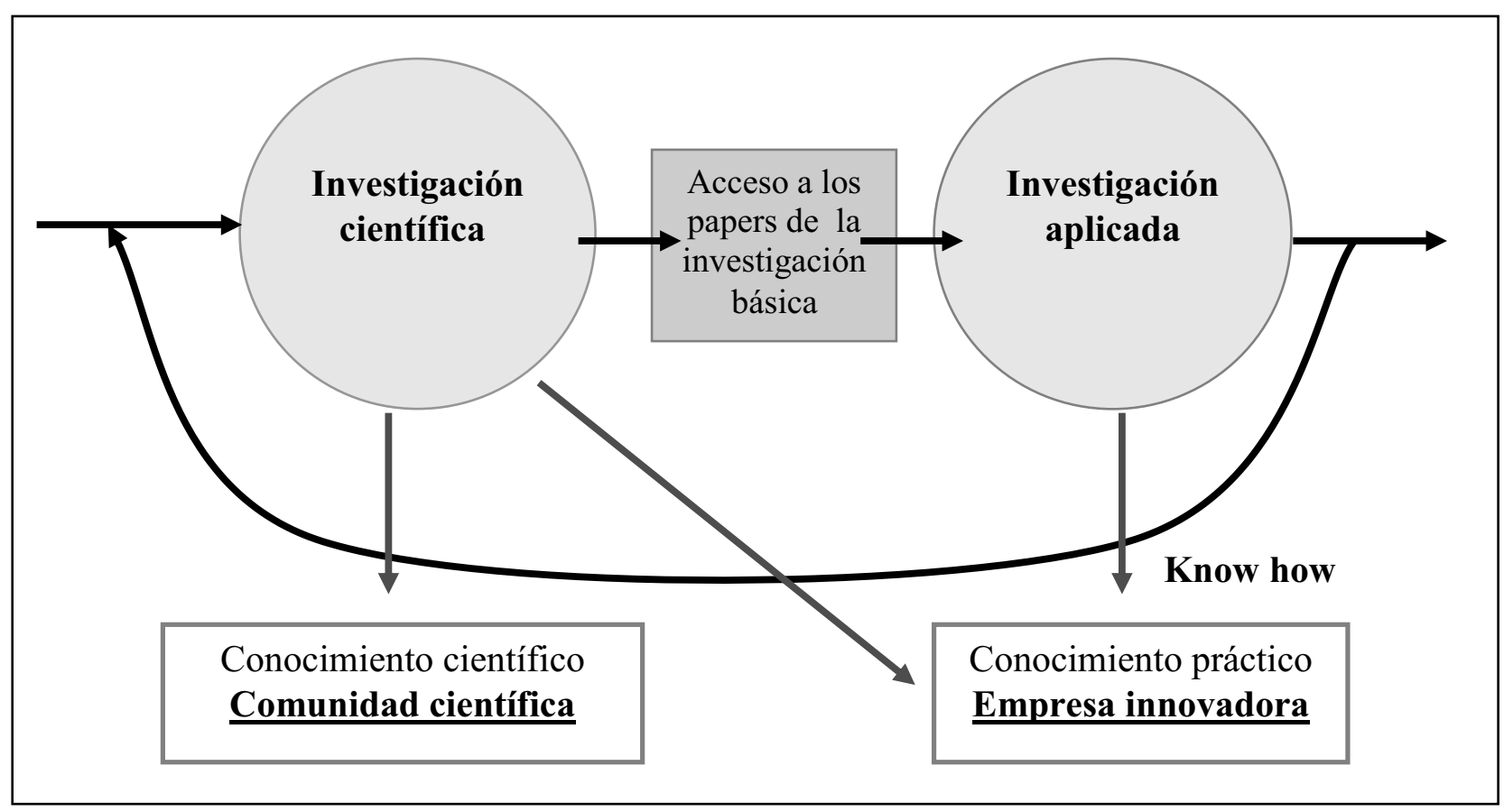

FUENTE: Elaboración propia. 
Figura N. $^{\circ}$ 3. Ciencia y Tecnología

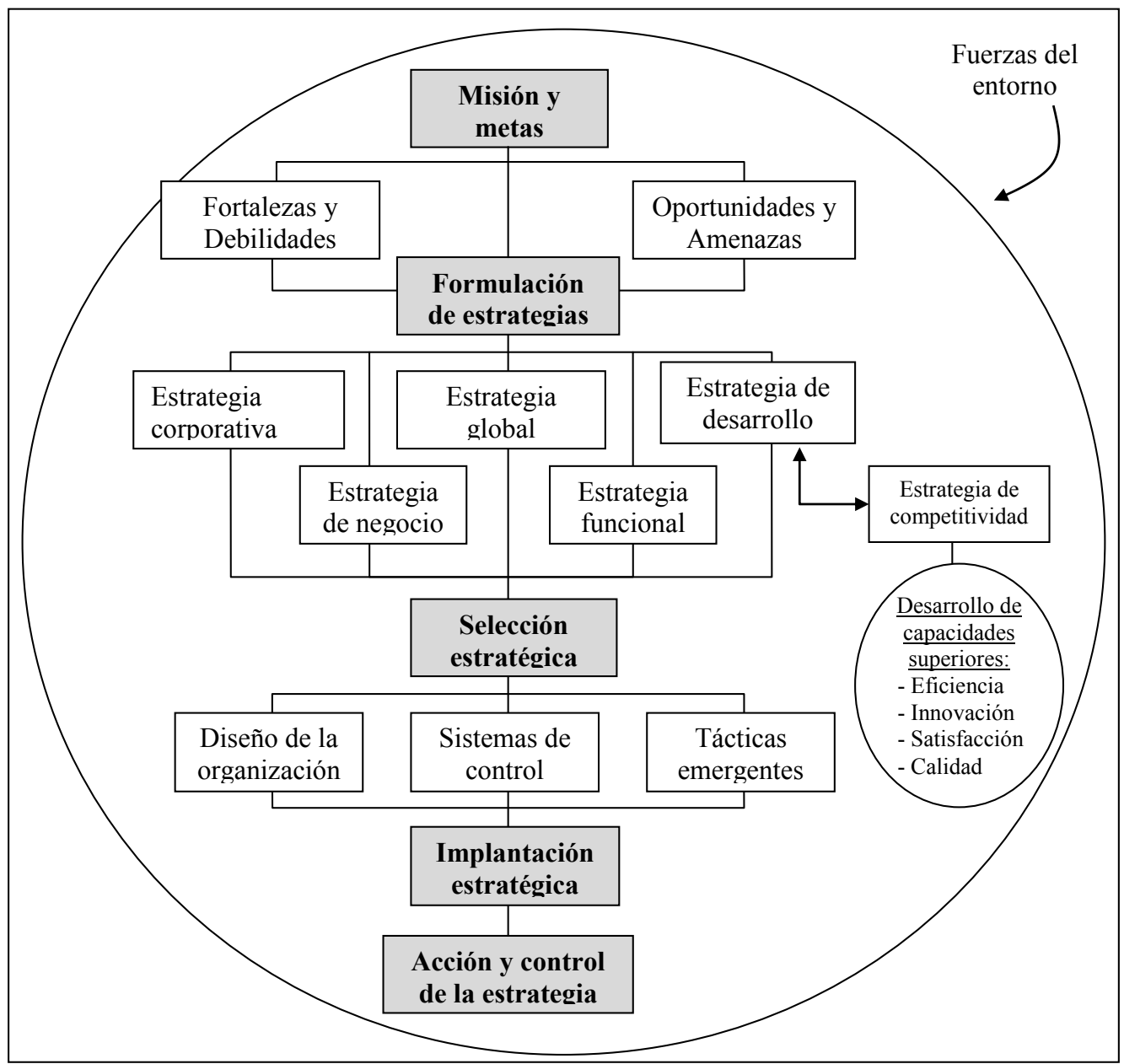

FuENTE: Linares, Carolina (2005). Nota técnica sobre innovación tecnológica.

nada, un momento de inspiración o mecanismos subconscientes desconocidos, que no se planean, administran ni sistematizan.

A partir de las experiencias de empresas innovadoras y dominantes, se ha iniciado la corriente que promueve que las mejores empresas han desarrollado una estructura que permite un flujo regular de innovaciones que aseguran la sostenibilidad empresarial.

\section{El diseño de las estrategias y la innovación}

En la empresa, este modelo de innovación tecnológica utilizando matrices, es una síntesis de la visión directiva, integrando la variable tecnológica en el planeamiento de los servicios (Figura N. ${ }^{\circ} 3$ ). Es una herramienta para la labor directiva, donde se descubren e integran, en forma sistemática e incremental, las necesidades no cubiertas y las oportunidades de innovación que se plasman en mejoras del servicio.
El proceso de administración estratégica se inicia con la definición de la misión, que se orienta a alcanzar metas dentro de una visión y un escenario de largo plazo. Se analiza internamente para identificar sus capacidades y debilidades y se explora externamente para percibir las oportunidades del mercado, luego se analizan y formulan las estrategias corporativas, globales, desarrollo, de negocio y funcionales, las que son sintetizadas por la dirección para seleccionar el arreglo estratégico que dirige la operación y resultados de la empresa (Hill \& Gareth \& Jones, 1996).

Dentro de la estrategia de desarrollo se considera la innovación, referida a la capacidad de crear y mantener fuentes de competitividad. Estas fuentes están estrechamente ligadas al cambio y mejora en las cuatro dimensiones del mundo de la empresa (estructural, de procesos, personas y tecnológica). El cambio permanente permite que las empresas desarrollen las cuatro capacidades requeridas para alcanzar y mantener ventajas competitivas: innovación, calidad, eficiencia y satisfacción del cliente. 
Figura N. ${ }^{\circ}$ 4. El modelo de innovación en el diseño estratégico

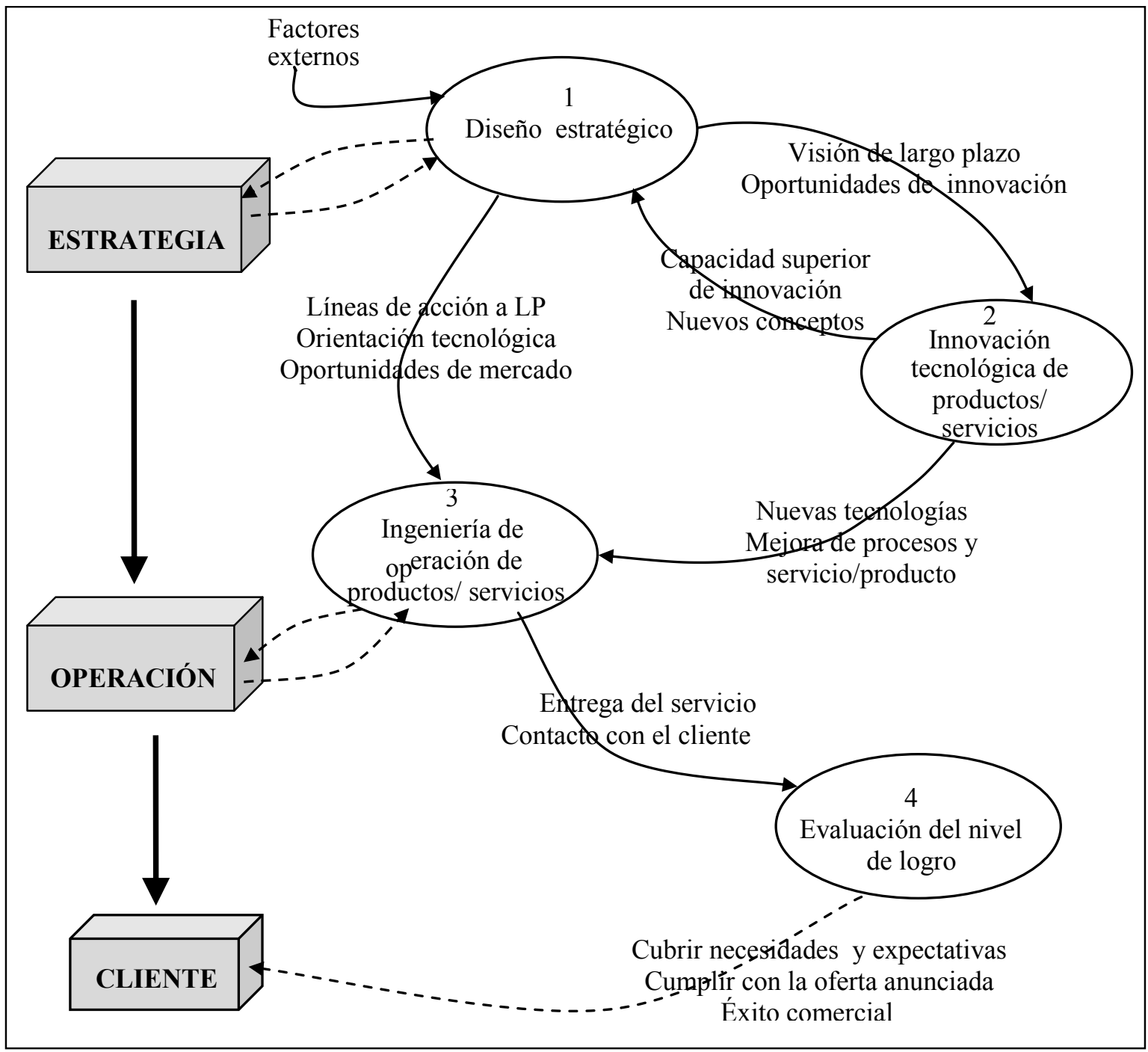

FUENTE: Ibíd.

La capacidad de innovación implica que la estrategia de desarrollo se alinea con las demás estrategias de la compañía, de manera que los recursos se concentran en acelerar las actividades que se orientan a buscar y encontrar las innovaciones emergentes rentables, las que surgen con el cambio en las tendencias de los mercados locales y globales, aumento poblacional, quiebres tecnológicos y nuevas preferencias de los clientes.

La innovación superior en las empresas, se basa en cuatro premisas:

- La inclusión de criterios de desarrollo en las 4 dimensiones, dentro del proceso de planeamiento y administración estratégica, que devienen en capacidades superiores.

- El diseño y la estructuración de una consistente y sostenida capacidad innovadora.

- Un modelo operativo de ingeniería y operación que aplique la capacidad innovadora en el funcionamiento de la empresa en marcha.

- Retroalimentación para verificar el grado de desarrollo de las capacidades superiores.

\section{La innovación dentro del diseño estratégico}

La empresa dominante del presente siglo, la que se está convirtiendo en motor del crecimiento en las economías menos desarrolladas, es una empresa innovadora. La innovación considera factores tecnológicos, factores de proceso y administrativos y factores humanos, dentro de los cuales han de plantearse las oportunidades de cambio, elaboradas mediante la estrategia de innovación.

Las empresas exitosas han de ser organizaciones innovadoras, que perciben y aprovechan las oportunidades y el cambio. Esta innovación debe consi- 
Figura 5. MÓDULO 1: Diseño estratatégico de la innovación

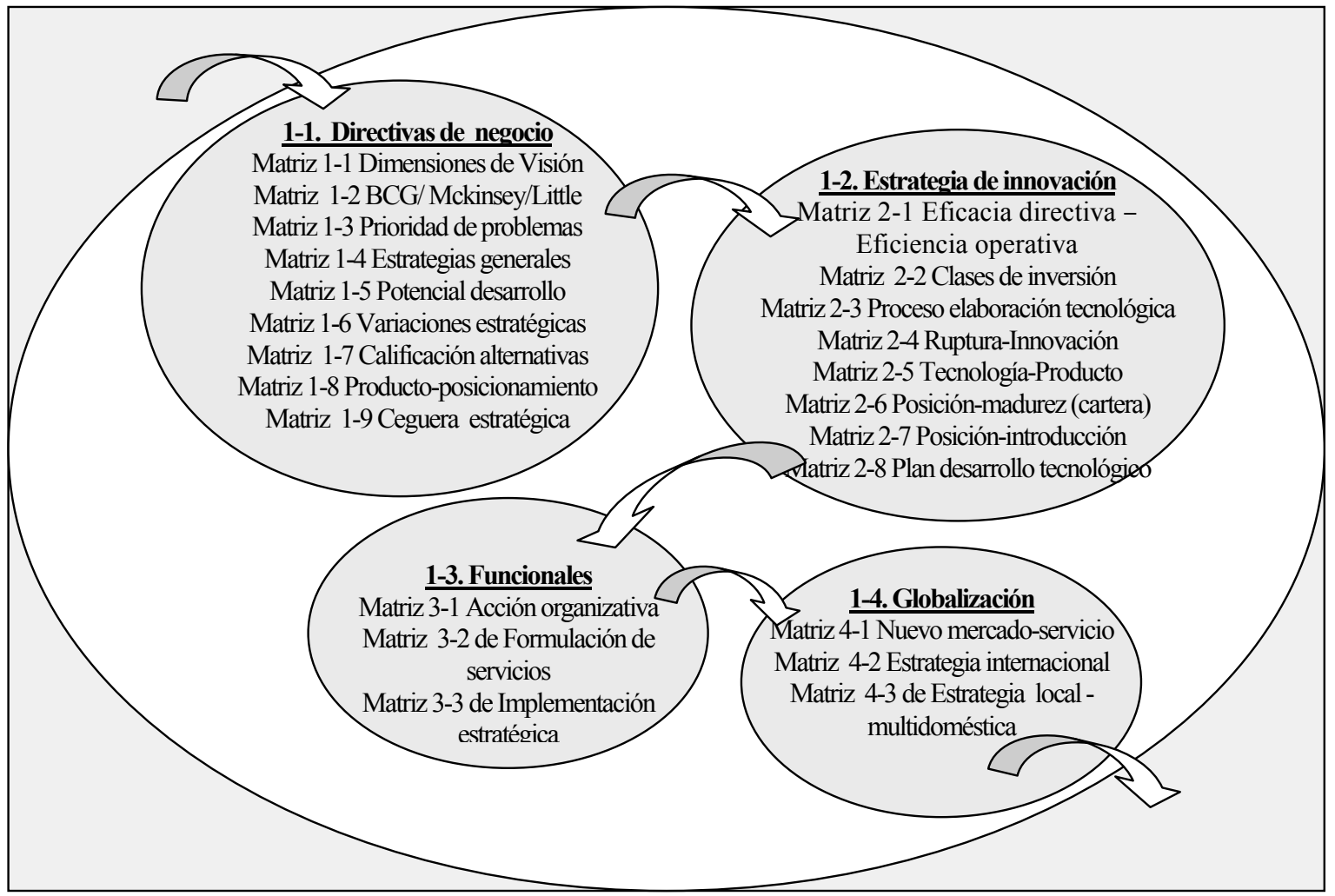

FUENTE: Elaboración propia.

derar la composición y las fuerzas de los factores ambientales, del proceso de negocio, del funcionamiento administrativo y del ambiente humano.

Esta innovación, para ser eficaz, debe iniciarse con preguntas estratégicas:

- ¿Qué es nuestra empresa y a dónde vamos?

- ¿Cuál es la tendencia en las variables sociales y tecnológicas? ¿Pueden ser fuente de futura ventaja competitiva?

- ¿Cómo ayuda la innovación tecnología para mejorar el servicio que brindamos?

- ¿Cómo se ha de incorporar en forma sistemática, la variable del cambio e innovación en el planeamiento de largo plazo?

- ¿Cuándo y cómo introducir los cambios e incorporarlos en los productos y servicios?

- ¿Cuál es la expectativa del cliente?

A partir de las respuestas, se plantearían las actividades de desarrollo y operación de los procesos productivos, considerando cuatro grandes procesos: planeamiento estratégico, diseño de la innovación, ingeniería de operación y la satisfacción del cliente (Linares, 2005).

El modelo de innovación consta de cuatro grandes subprocesos o módulos, cuyas interrelaciones bá- sicas se presentan en la Figura N.$^{\circ} 4$. Estos módulos se refieren al diseño estratégico de la innovación, la mejora de procesos y servicios aprovechando los cambios tecnológicos y del conocimiento, la ingeniería de la operación de los productos y servicio y la evaluación del logro de la innovación, mediante la calificación y seguimiento de las variables de control.

\section{EL MODELO DE INNOVACIÓN EN LA EMPRESA}

El modelo consta de cuatro procesos con sus respectivos subprocesos. El modelo implica una labor de diseño con múltiples dimensiones y variables de creciente complejidad conceptual. El uso de herramientas gráficas (o matrices) facilita la síntesis estratégica y permite percibir oportunidades de mejora y para el diseño de los cambios. Las matrices elaboradas siguen una secuencia top-down, donde se van obteniendo los elementos necesarios para el diseño estratégico de la innovación.

\section{El proceso 1 de diseño estratégico}

El planeamiento estratégico de la empresa recibe información de mercado y tecnológica, que permite delinear la orientación en el diseño de servicios y en 


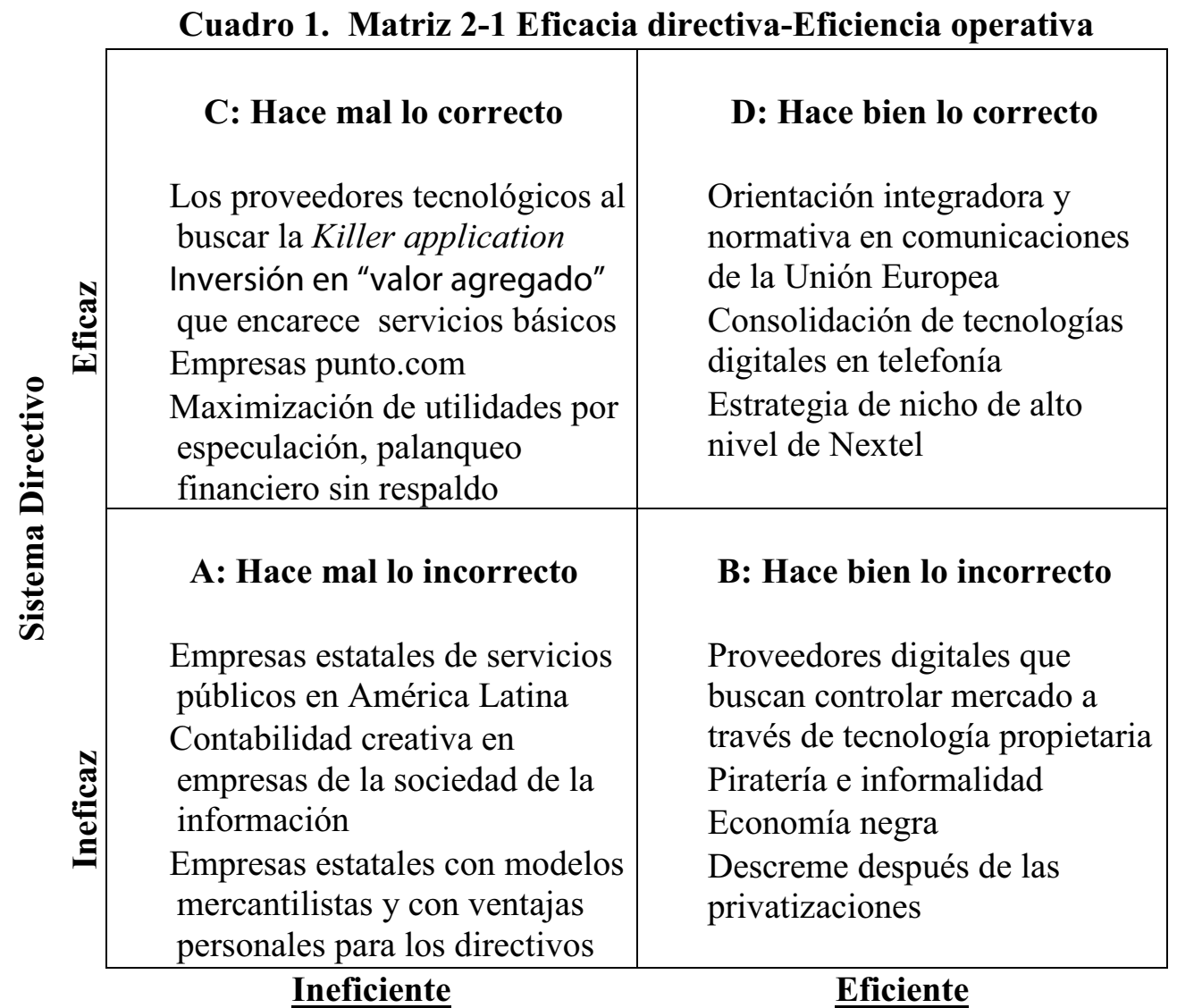

Fuente: Elaboración propia. Basado en Picazo y Martínez, 1992.

la operación misma. Consta de cuatro módulos que se desarrollan mediante las matrices que se indican en la Figura N. ${ }^{\circ} 5$.

A modo de ejemplo, se muestra la matriz 2-1 de Eficacia directiva-Eficiencia operativa, que integra los conceptos de metas y operación, para los dos grandes sistemas de dirección y operación empresarial. Es una medida de excelencia empresarial:

- Excelencia = Eficacia en logro de objetivos + eficiencia en costos de actividades.

- Excelencia = brecha de decisiones + brechas de tareas.

A través de dos ejes de coordenadas se definen cuatro cuadrantes referidos a la acción y la dirección. El eje " $x$ " se refiere al sistema operativo en sus opciones de eficiencia o ineficiencia en la ejecución de las tareas de obtención de recursos, operación y comercialización. El eje "y" se refiere al sistema directivo midiendo el logro de objetivos y metas. Ayuda a descubrir fuerzas y debilidades en la ejecución (qué tan bien funciona la organización) y dirección (qué buenas decisiones se toma), como se observa en la matriz del Cuadro N. ${ }^{0} 1$.
Cuadrante A: Ejemplo de malas decisiones y mala operación es la empresa privada que "infla" el valor de sus activos mediante manejo de la contabilidad. Cuadrante B: Las empresas de malas decisiones, aunque funcionen bien, generan problemas ético-legales o externalidades (sobrecostos), un caso es el proveedor de tecnología propietaria que restringe las posibilidades de operación de sus clientes, lo que aumenta sus ganancias en el corto plazo pero en el largo plazo tiende a cero.

Cuadrante C: Empresas con buenas decisiones pero en la dirección equivocada; ejemplos son las empresas que conformaron la burbuja punto.com y las que surgen con las opciones de la nueva economía

Cuadrante D: El mejor ejemplo de hacer bien lo correcto es la tecnología de comunicaciones que insume gran cantidad de recursos en la preparación, diseño y estandarización y su operación deviene en eficiente, de manera que subsidia los productos de los sectores de entretenimiento y mass-media. 
Figura 6. MÓDULO 2: Innovación tecnológica de productos/servicios

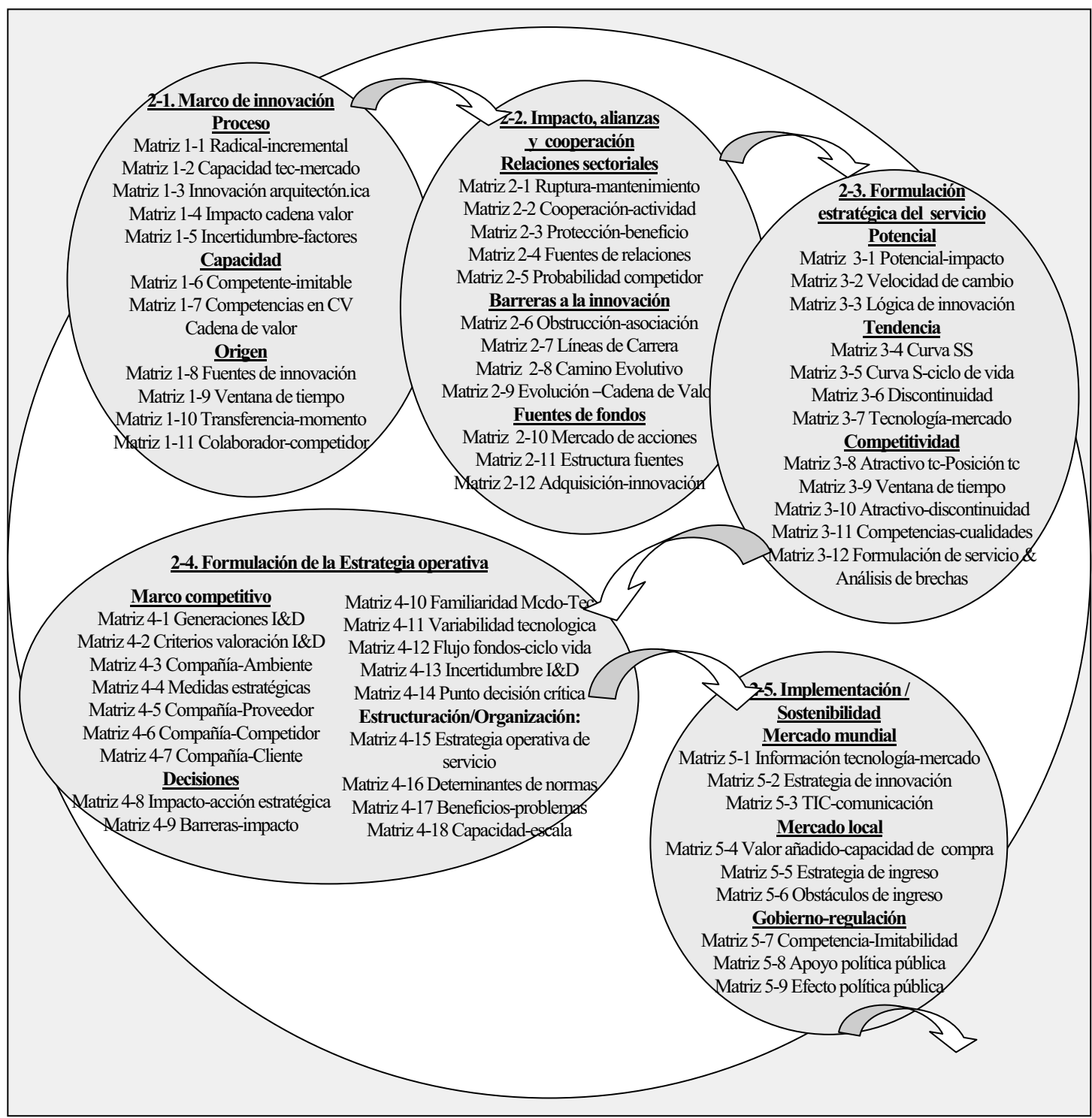

FUENTE: Elaboración propia.

\section{El proceso 2 de innovación tecnológica de productos/servicios}

Para desarrollar la innovación, como proceso de creación de nuevo conocimiento, se requiere talento para detectar las oportunidades de renovación, capacidad analítica para diferenciar los elementos y capacidad de síntesis para la sistematización y puesta en práctica. Consta de cinco subprocesos que se presentan en la Figura N. ${ }^{\circ} 6$.

El marco de la innovación se refiere a la perspectiva asumida para estudiar los factores de innovación y la capacidad de diferenciación o bajo costo y entender las fuentes de innovación.
Para fines explicativos mostramos el uso de la matriz 1-1 Radical-incremental, que identifica los criterios que se emplean para que las empresas establecidas superen a los competidores antiguos o los nuevos sustitutos, intenta mostrar las consecuencias posibles de un cambio incremental o radical, acelerado por el conocimiento del mercado de la empresa.

Se estructura en los ejes $x-y$. El eje $x$ se refiere a la innovación tecnológica en sus opciones: implantación suave e incremental o implantación radical con ruptura de las reglas vigentes. El eje " $y$ " se refiere a los dos tipos de empresa participantes: las establecidas con conocimiento del mercado y las nuevas que no conocen el mercado y no poseen prejuicios ni miopía frente a los eventos. 


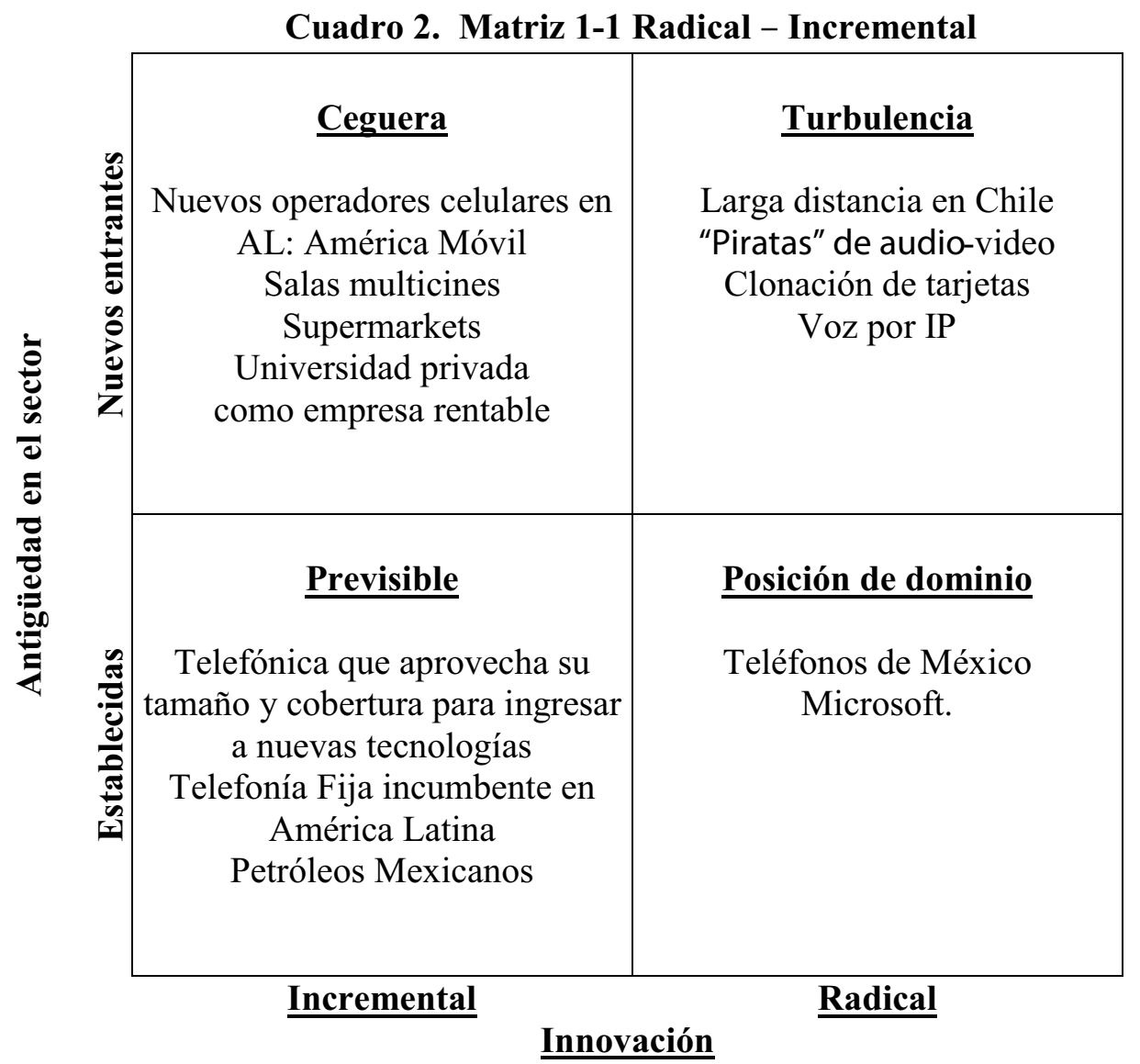

Fuente: Elaboración propia. Basado en Escorsa-Valls, 2001.

En el Cuadro N. ${ }^{\circ} 2$ se presentan los cuadrantes de la matriz:

- Previsible. Los ya establecidos pueden ganar debido a su conocimiento del mercado. Es el caso de la empresa Telmex que controla el mercado independiente de la tecnología.

- Posición de dominio. Los establecidos pueden mantener poder de mercado si son los primeros en aplicar nuevas tecnologías diferenciadoras.

- Ceguera. Los nuevos entrantes ganan cuando el conocimiento de mercado se torna obsoleto. Ejemplo son las grandes salas de pantalla panorámica que han sido superadas por las salas multicines, nuevas universidades privadas desplazan a las públicas.

- Turbulencia. Situación crítica si no se asume un liderazgo claro, al no identificarse a la empresa dominante, puede surgir "guerra" de mercado que descontrolaría el funcionamiento del sector. Ejemplo: el sector informal de videopirata.

\section{EI Proceso 3 de ingeniería de operación de productos/ servicios}

Este proceso plantea la innovación en la operación diaria de la organización productiva o de la empresa de ser- vicios, desde el objetivo, los factores externos que inciden en la prestación, la mejora, y luego la comercialización. Sus subprocesos se muestran en la Figura N ${ }^{\circ} 7$.

Como ejemplo se muestra el cuadro 3, la matriz 3-3 Gestión tecnológica de productos-servicios que considera la relación entre inversión en tecnologías y la gestión del servicio con la capacidad para atender a diferentes segmentos. A nivel estratégico define el énfasis de la empresa: orientado a las personas u orientado a la inversión, a fin de diseñar las funciones y tareas, acordes al perfil del cliente objetivo. Lleva a términos operacionales, la estrategia tecnológica general y sienta las bases del diseño operativo del producto o servicio.

Esta matriz contempla la masificación del servicio en el eje $x$, y la intensidad tecnológica en el eje $y$. Con estas dos dimensiones se definen las cuatro áreas de la matriz, cada una indica el grado de participación de nuevas tecnologías en el proceso de prestación de servicios y las implicaciones derivadas de su adopción.

A: Servicio a la medida. En estas empresas existe alto potencial de uso de tecnologías avanzadas, 
Figura N. ${ }^{\circ}$ 7. Módulo 3: Ingeniería de la operación de productos

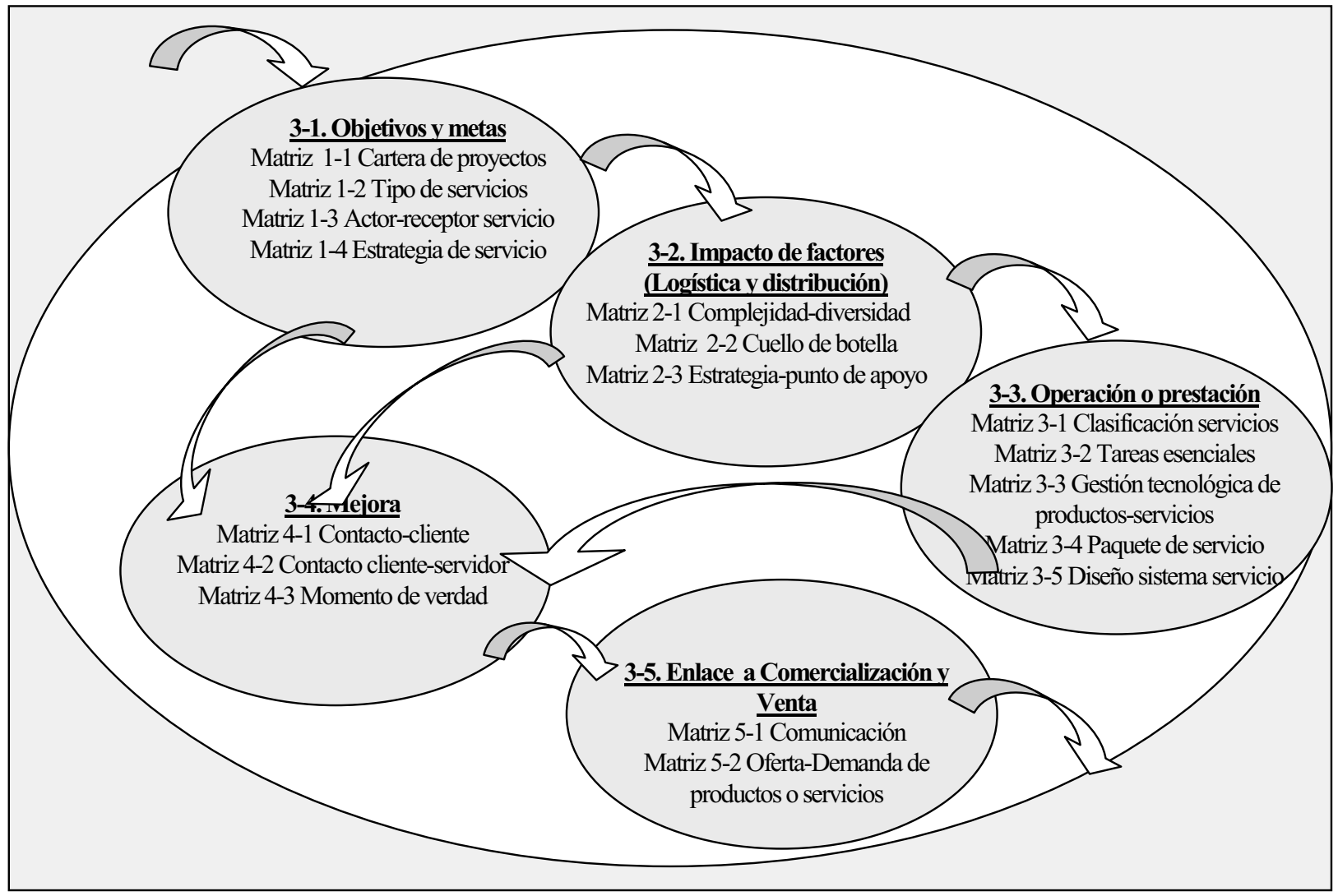

FUENTE: Elaboración propia.

Cuadro 3. Matriz 3-3 Gestión tecnológica de Producto-Servicio

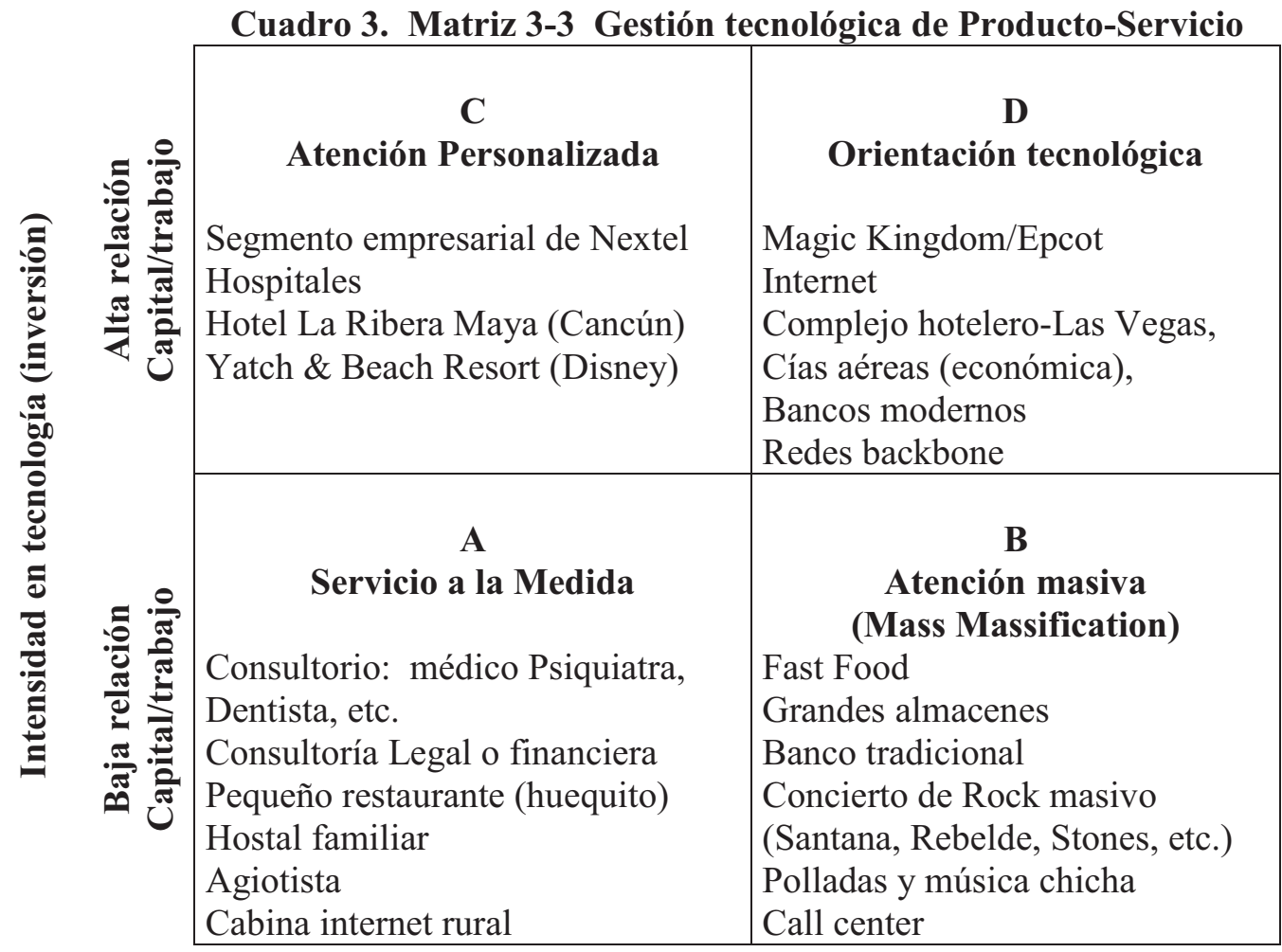

Fuente: Elaboración propia. Basado en Martínez, 1991. 
Figura 8. Módulo 4: Evaluación del nivel de logro

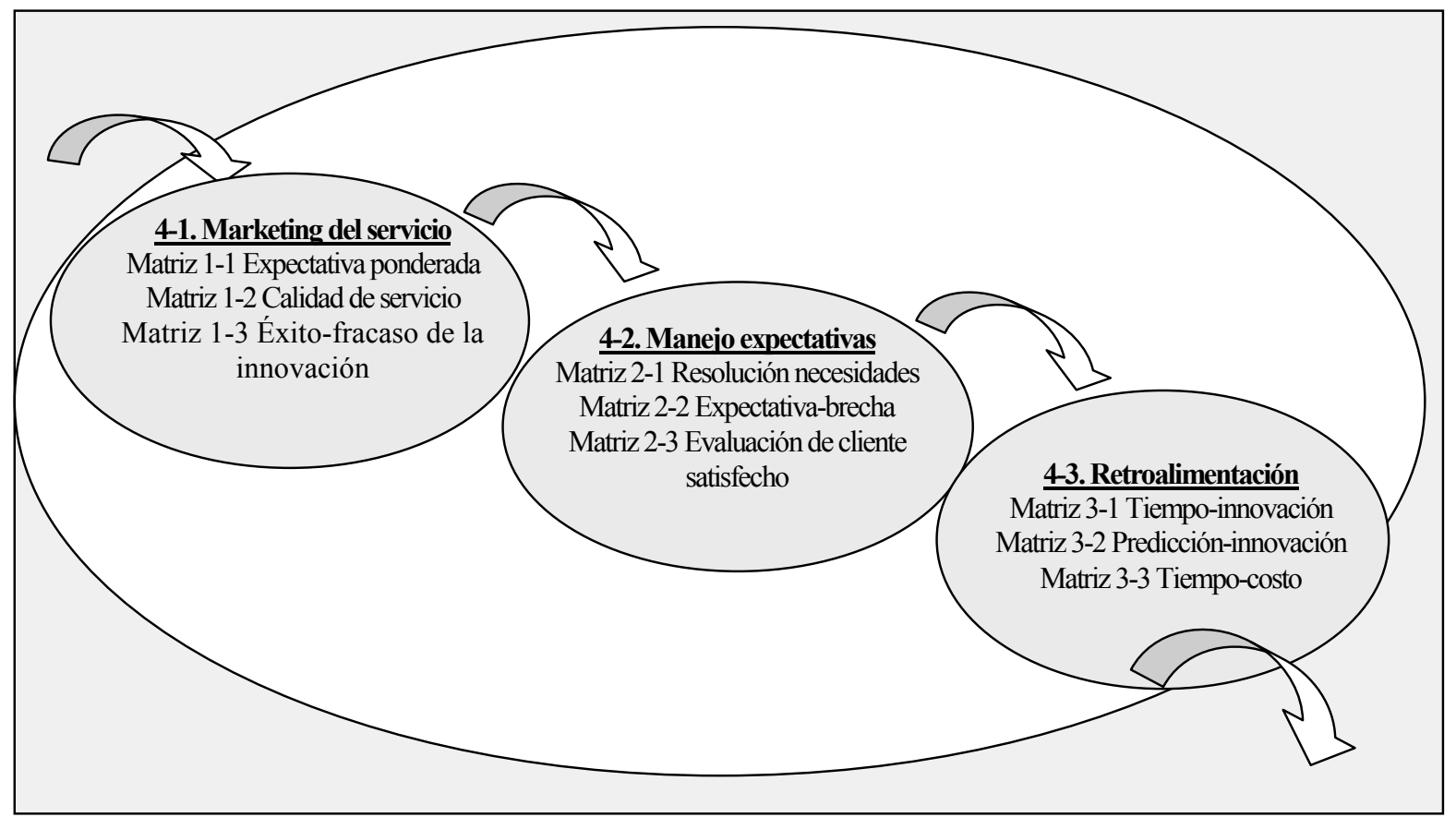

FUENTE: Elaboración propia.

como internet, que permite la conexión a bases de datos y la automatización de oficinas, creando oportunidades de diversificación, aunque manteniendo la atención individualizada.

B: Mass massification. Estas empresas pueden tipificarse de prestación masiva de servicio, donde la preocupación directiva estará más enfocada en el marketing global de los servicios y la capacitación de personal generalista.

C: Atención personalizada. La mayor interacción con el cliente puede limitar la introducción de tecnologías avanzadas, ya que el servicio se sustenta en el conocimiento, pero careciendo de especialistas técnicos. La dirección tiende a desplazarlas hacia la inversión en tecnologías avanzadas para reducir la relación de "dependencia" con el cliente, economía en costos y mayor control de la demanda de los servicios.

D: Orientación tecnológica. Considera los servicios intensivos en tecnología y bajo grado de interacción e individualización del cliente; son empresas fuente y destino de innovaciones tecnológicas. El personal es especialista de alto nivel y diverso, enfocados en la tecnología, lo que permite el seguimiento de avances, estar a la vanguardia en la adopción de tecnologías avanzadas y poca dependencia de los proveedores. Los directivos asumen decisión sobre la adopción de tecnologías, a la vez que controlan los avances tecnológicos de otros, tie- nen fuerte influencia en proveedores relacionados a su empresa.

\section{El proceso 4 de Evaluación del nivel de logro}

El nivel de logro se relaciona con la satisfacción del cliente. Este proceso sintetiza la finalidad de la prestación de servicios y guarda estrecha relación con el marketing en su rol de hacer conocer el servicio, identificar necesidades, expectativas del cliente y la retroalimentación para verificar la brecha entre lo esperado y lo brindado. La Figura N ${ }^{\circ} 8$ muestra los subprocesos.

La matriz 1-3 Éxito-fracaso de la innovación, que relaciona el proceso de innovación con los resultados reales en el mercado. El eje " $x$ " se refiere al grado de novedad de los productos lanzados y el eje "y" se refiere a los resultados de la innovación.

\section{En el cuadro 4 se presenta un ejemplo aplicativo de esta matriz.}

A: Innovador-Fracaso. Los factores de fracaso de un nuevo producto son: a) tangibles: poca utilidad, baja calidad, precio inadecuado, poca publicidad, presentación inadecuada, b) intangibles: organización, inoportuno, mal estudio de mercado, decisiones emocionales.

B: Seguidor-Fracaso. Aparte de las fallas del innovador, el fracaso deriva de deficiencias en producir 


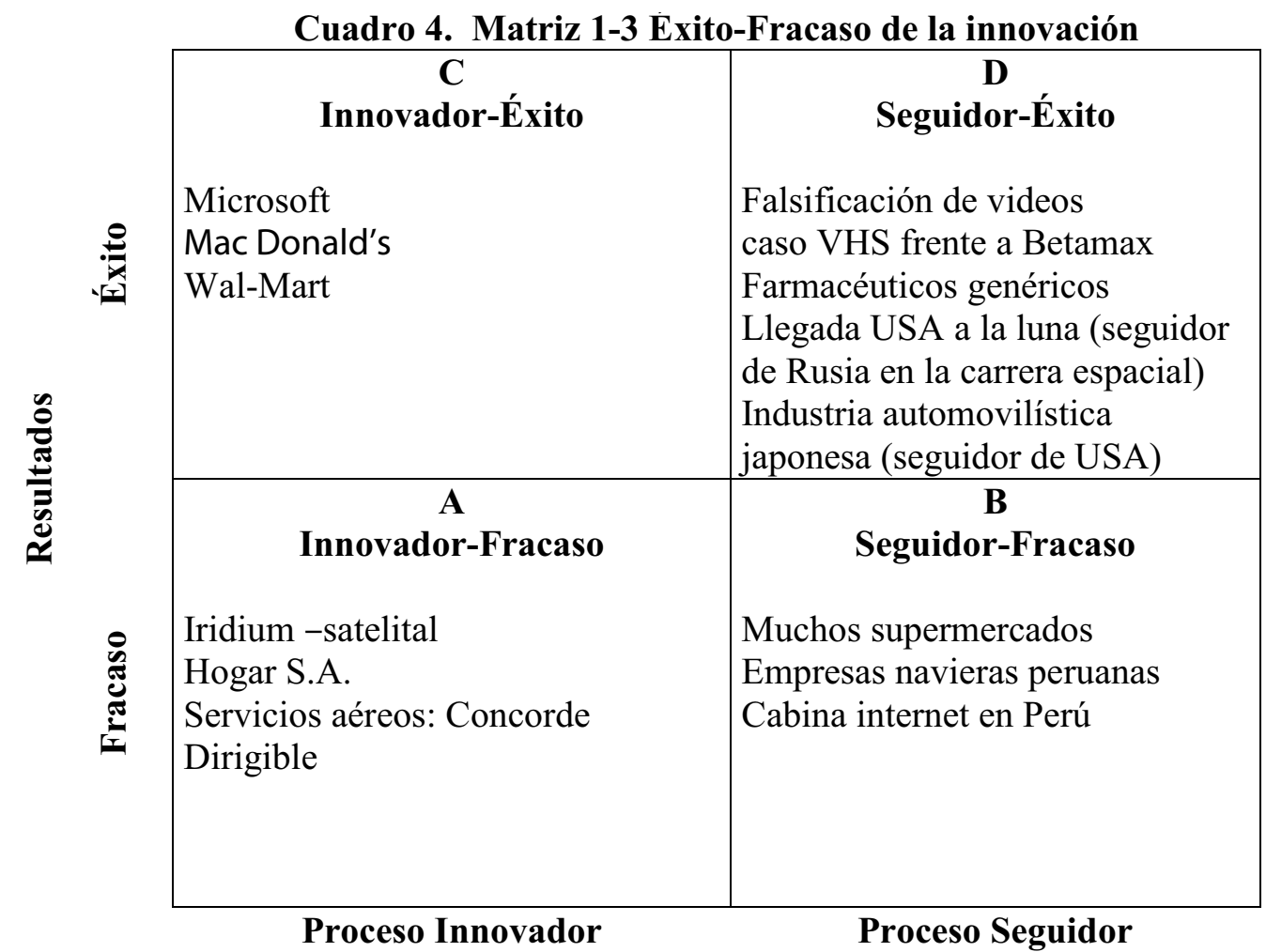

Fuente: Elaboración propia. Basado en Escorsa-Valls, 2001.

ventajas únicas y falta de sinergias con las actividades habituales de la empresa.

C: Innovador-Éxito. El éxito de un nuevo servicio está estrechamente ligado con las actividades desde la idea hasta la comercialización. Estas actividades previas comprenden: aspectos conceptuales (técnica de servicio), aspectos de mercadeo (aceptabilidad, percepción), aspectos industriales (materiales, proveedores, proceso), existencias, diseño, negociaciones.

D: Seguidor-Éxito. En algunos casos el seguidor supera al primero. Cuando la innovación es demasiado arriesgada, el seguidor aprovecha las fallas del innovador. El innovador tiene altas posibilidades de perder si no se cumplen tres requisitos: a) No tiene asegurada la propiedad del producto y el grado de imitabilidad es elevado, b) el producto o servicio no llega a ser aceptado como el paradigma de la industria y c) existen deficiencias empresariales como: mercadeo, asistencia postventa, formación.

\section{CONCLUSIONES}

La innovación es la capacidad empresarial de mejorar los procesos de operación de la empresa, aprovechando las ventajas que brinda la tecnología. Innovación se define como una invención que se comercializa con éxito en el mercado.
Antiguamente, se consideraba que la innovación es un proceso irregular, fruto de una idea iluminada, un momento de inspiración o mecanismos subconscientes desconocidos, que no se planean, administran ni sistematizan. Pero, la evidencia muestra que las mejores empresas dominan sus mercados porque desarrollan procesos y estructuras que permite el flujo regular de innovación tecnológica y comercial, asegurando la sostenibilidad empresarial de largo plazo.

Es sustantivo el aporte de la innovación para la supervivencia y éxito empresarial, ya que condiciona la calidad y costo de los productos y servicios, determina la capacidad competitiva presente y potencial, influye en el aumento de la participación de mercado, lo que se refleja en los resultados financieros y en el valor de la empresa.

En el proceso de innovación intervienen actividades científicas diversas, cuestiones organizativas, financieras y comerciales; pero la tecnología es la variable que ejerce mayor impacto para el éxito en el nuevo entorno de negocios.

Ha surgido el nuevo paradigma que señala que la mejor empresa es la empresa innovadora que evoluciona mediante la adopción de nuevos procesos de fabricación de productos o prestación de servicios, a un ritmo cada vez mayor debido al aumento del conocimiento, nuevas tecnologías, la globalización de la eco- 
nomía, mayor tamaño de los mercados y la masificación con ofertas cada vez más personalizadas.

La aceleración del cambio en los ambientes económico, social, político y tecnológico, exige el cambio de paradigmas en la mente de los directivos:

- Visión trascendente, sentido de misión y perspectiva de conglomerado que supere el ámbito del sector industrial, donde la variable innovación es parte del planeamiento, en un proceso simultáneo de diseño de la estrategia de negocio y la estrategia tecnológica.

- Esfuerzo sostenido de innovación en productos, procesos y servicios, enfatizando la innovación en tecnología, por su impacto en la ruptura de reglas competitivas.

- Velocidad de respuesta a los fenómenos mundiales y la demanda global, mediante el manejo de información enfocada en los mercados y los clientes.

\section{REFERENCIAS BIBLIOGRÁFICAS}

1. Abernathy y Clark (1985). "Mapping the winds of creative destruction". En: Research Policy, 14.

2. Afuah, Alan (1999). La dinámica de la innovación organizacional, Oxford University Press.

3. Band, William (1991). Creating Value for Customers, Moving From Talk to Action, John Willey \& Sons, Inc. New York.

4. Escorsa y Valls (2001). Tecnología e innovación en la empresa, 2001 ALFAOMEGA, S.A.
5. Hill, Gareth, Jones (1996). Administración Estratégica un enfoque integrado, McGraw-Hill Interamericana.

6. Kuhn, Thomas (1962). La Estructura de las Revoluciones Científicas, Fondo de Cultura Económica, México.

7. Linares, Carolina (2005). "Nota Técnica sobre innovación tecnológica en empresas de servicios", Universidad de Chile, Santiago de Chile.

8. Martínez, Ángel (1991). "Las implicaciones tecnológicas del sector servicios". En: Alta Dirección, 158, julio-agosto.

9. Picazo y Martínez (1992). Ingeniería de Servicios, McGraw-Hill/Interamericana.

10. Porter, Michael (1999). Ser competitivo, Deusto, España.

11. Rousell, Saad, Erickson (1991). Third Generation R\&D, Harvard Business School Press, Boston.

12. Tushman y Rosenkopf (1992). "Organizacional determinants of technological change: Towards a sociology of technological evolution". En: Research in Organizational Behaviour, 14.

13. Stern, Carl \& Deimler, Michael (2006). The Boston Consulting Group on Strategy, John Wiley \& Sons, Inc. 